\title{
Impact of salpingectomy for ectopic pregnancy on the ovarian response during IVF stimulation
}

\author{
Camille Gay $^{\mathrm{a}, *}$, Jeanne Perrin ${ }^{\mathrm{a}, \mathrm{c}}$, Blandine Courbiere ${ }^{\mathrm{a}, \mathrm{c}}$, Florence Bretelle ${ }^{\mathrm{c}, \mathrm{d}}$, \\ Aubert Agostini ${ }^{\mathrm{b}, \mathrm{c}}$ \\ a Clinico-Biological Center of Medical Assistance for Procreation, AP-HM La Conception, 147 bd Baille, 13005, Marseille, France \\ ${ }^{\mathrm{b}}$ Gynecology and Obstetrics Center, AP-HM La Conception, 147 bd Baille, 13005, Marseille, France \\ ' Aix Marseille Université, CNRS, IRD, Avignon Université, IMBE UMR 7263, 13397, Marseille, France \\ d Gynecology and Obstetrics Center, AP-HM Hôpital Nord, chemin des Bourrely, 13015, Marseille, France
}

Keywords:

Salpingectomy

Ovarian response

IVF

Fertility

\begin{abstract}
A B S T R A C T
Introduction: Ovarian reserve is a major prognosic factor for Medical Assisted Procreation. Tubal surgery, realised close to mesosalpinx and ovarian vascularization, could impare ovarian function.

However, salpingectomy is currently used to treat ectopic pregnancies or hydrosalpinx before IVF attempt. Disponible studies on this subject are unclear. The aim of this study is to evaluate the impact of salpingectomy for ectopic pregnancy on the ovarian response during IVF attempt.

Material and methods: It was a single center comparative study. Included patients were the one receiving oocyte puncture for IVF attempt, with a history of unilateral tubal surgery: salpingectomy for ectopic pregnancy. We conducted a case-control study, comparing the sonographic parameters of the surgery ovary (case) to those of the safe ovary (control) during the first IVF attempt after salpingectomy. The ovarian sonographic response was evaluated according to the follicular antral count on day 3 and the sonographic follicular count on trigger day.

Results: 55 patients were included. There was no significant difference in the number of recruited follicles on the operated side versus control side ( $p=0.85$ for $>14 \mathrm{~mm}$ follicles, $p=0,46$ for 10 to $14 \mathrm{~mm}, p=0,52$ for total amount of recruited follicles). There was no significant difference for the follicular antral count neither $(\mathrm{p}=0.79)$.

Discussion: In our population, there was no significant difference in the sonographic ovarian response to IVF stimulation between the ovary on the operated side and the control ovary among patients treated by unilateral salpingectomy for ectopic pregnancy.
\end{abstract}

\section{Introduction}

Increasingly more patients resort to medical assistance for procreation Medical Assisted Procreation (MAP). Ovarian reserve, and thus ovarian integrity, is one of the principal prognostic factors. Many of our patients have a history of tubal surgery, some of them having been performed for ectopic pregnancies, for whom salpingectomy is the benchmark treatment when the contralateral fallopian tube is healthy [1,2]. In addition, we know that the presence of a hydrosalpinx reduces pregnancy rates in MAP [3], and salpingectomy is recommended in this situation to improve implantation rates [4-6]. Surgical impact on ovarian reserve and function must be known to determine indications, benefits and risks.

\footnotetext{
* Corresponding author.

E-mail address: camille.gay@ap-hm.fr (C. Gay).
}

Ovarian vascularization is provided by the ovarian and uterine arteries and is close to the fallopian tube and mesosalpinx. Thus, fallopian tube surgery may impair ovarian vascularization and function. This outcome has been confirmed with animal studies [7], but the result from human studies remains unclear.

Available studies on the impact of salpingectomy on the ovarian response during IVF are contradictory $[8,9]$. These contradictions are partly because the impact might be different according to the indication of the surgery [9]. The access to uniform studies, the evaluation of the impact on the ovary according to the indication of the salpingectomy and the use of direct parameters for evaluation of the ovarian response are necessary.

To study the direct impact on the ovary, it would be interesting to study sonographic follicular modifications during IVF stimulation. Only 4 studies have analysed this parameter; Tal and Xi's studies $[10,11]$, which did not find a reduction in the homolateral ovarian response among respectively 26 and 76 operated patients, 
and Gelbaya and Orvieto's studies, which highlighted a decrease in ovarian response among 40 and 15 patients per study12,13. However, the included patients had various salpingectomy indications, and that is why it would be interesting to evaluate the sonographic follicular modifications among patients treated by salpingectomy for the same indication. The principle aim of our study is to evaluate the sonographic impact on the homolateral ovarian response after salpingectomy for ectopic pregnancy (EP) during IVF stimulation.

\section{Material and methods}

This was an observational single centre comparative study that was conducted in the clinicobiological MAP centre of the university hospital of Marseille, France, between 1 January 2008 and 31 March 2018. Eligible patients were those treated for IVF +/- ICSI in the centre who received oocyte puncture during this period, whether or not embryo transfer was performed. Included patients were those who had a history of unilateral tubal surgery: salpingectomy for EP before IVF attempt. The excluded patients were those who received an other type of tubal surgery, bilateral tubal surgery or ovarian surgery.

We conducted a case-control study, comparing the sonographic parameters of the surgery ovary (case) to those of the safe ovary (control) during the first IVF attempt after salpingectomy for EP. The ovarian sonographic response was evaluated according to the follicular antral count on day 3 and the sonographic follicular count (divided in more than $15 \mathrm{~mm}$ and 10 to $14 \mathrm{~mm}$ ) on trigger day. Sonography were realised with the same ultrasound scanner, in our center.

Clinico-biological and stimulation-related data were extracted from the medical file: age, smoking status, BMI, IVF indication, and ovarian reserve parameters (AMH, follicular antral count FAC, and FSH on day 3). Salpingectomy information was collected: indication, side, time between surgery and IVF attempt, and laparoscopy or laparotomy. Finally, IVF attempt information was collected: number and size of recruited follicles visible with sonography on each ovary, day of the ovulation trigger, plasmatic oestradiol on the day of trigger, total gonadotrophin dose, protocol (short, long, antagonist), number of oocytes collected and number of mature oocytes.

The chosen threshold for follicular size (between 10 and $14 \mathrm{~mm}$ and more than $14 \mathrm{~mm}$ ) is the usual threshold used in published studies $[10,12,13]$. Our study received favourable opinion from the Aix Marseille University ethics committee (2018-27-09-002). Qualitative values are compared with test or Fishers exact test. The live birth rate and secondary outcome measures were compared between the operated side and the safe control side. Qualitative values were formulated as headcount and percentage, and continuous variables as averages with standard deviation (or median and min max). The results were given as the odds ratio with a confidence interval of $95 \%$. Analyses were performed with Microsoft Excel 2010. The significance level was established at $5 \%$.

\section{Results}

During this period, 55 patients were included. Patients were aged an average of 34 years $( \pm 5)$.

Ovarian reserve parameters on day 3 were FSH 7.5 UI/L $( \pm 2.3)$, AMH $3.5 \mathrm{ng} / \mathrm{ml}( \pm 2.7)$, and FAC $16.2( \pm 10.5)$. A total of 41 patients (75\%) had a tubal indication for IVF. Data concerning IVF follicular response were available for all patients. Data according to follicular antral count were available for $46 / 55$ patients (84\%), and data concerning the surgery approach was available for $19 / 55$ patients (35\%).
Table 1

Clinico-biological Characteristics.

\begin{tabular}{|c|c|}
\hline \multicolumn{2}{|l|}{ Variables : $(\mathrm{N}=55$ patients $)$} \\
\hline Age (years) & $34 \pm 5$ \\
\hline \multirow[t]{3}{*}{ Smoking } & Active 11 (20\%) \\
\hline & Former 7 (13\%) \\
\hline & Never 37 (67\%) \\
\hline BMI $\left(\mathrm{kg} / \mathrm{m}^{2}\right)$ & $23 \pm 4.3$ \\
\hline Right Salpingectomy & $35(64 \%)$ \\
\hline Left Salpingectomy & $20(36 \%)$ \\
\hline $\begin{array}{l}\text { Delay between IVF } \\
\text { attempt and } \\
\text { salpingectomy (years) }\end{array}$ & $3.3 \pm 3.8$ \\
\hline \multirow{2}{*}{$\begin{array}{l}\text { Surgical Technic ( } 19 \\
\text { patients) }\end{array}$} & Laparoscopy 14 (74\%) \\
\hline & Laparotomy 5 (26\%) \\
\hline \multirow[t]{6}{*}{ IVF Indication } & Tubal cause 41 (75\%) \\
\hline & Ovulatory cause $1(2 \%)$ \\
\hline & Man cause $1(2 \%)$ \\
\hline & Poor Ovarian Response 6 (10\%) \\
\hline & Idiopathic Cause $2(4 \%)$ \\
\hline & Endométriosis 4 (7\%) \\
\hline $\begin{array}{l}\text { Poor Ovarian Response } \\
\qquad(\mathrm{AMH}<1 \mathrm{ng} / \mathrm{ml} \text { et } / \mathrm{ou} \\
\left.\text { AFA }<7^{\circ}\right)\end{array}$ & $18(33 \%)$ \\
\hline $\begin{array}{l}\text { Follicular Antral Account } \\
\text { (46 patients) }\end{array}$ & $16.2 \pm 10.5$ \\
\hline FSH on Day $3(\mathrm{UI} / \mathrm{L})$ & $7.5 \pm 2.3$ \\
\hline $\mathrm{AMH}(\mathrm{ng} / \mathrm{ml})$ & $3.5 \pm 2.7$ \\
\hline \multirow[t]{2}{*}{ IVF or ICSI } & FIV 50 (91\%) \\
\hline & ICSI $5(9 \%)$ \\
\hline \multirow[t]{3}{*}{ Stimulation Protocol } & Long 25 (45.5\%) \\
\hline & Short $14(25.5 \%)$ \\
\hline & Antagonist 16 (29\%) \\
\hline Gonadotrophin (UI/L) & $2077 \pm 760$ \\
\hline $\begin{array}{c}\text { Plasmatic Estradiol on } \\
\text { Trigger Day }(\mathrm{pg} / \mathrm{ml})\end{array}$ & $2427 \pm 1246$ \\
\hline Trigger Day & $10.8 \pm 2.1$ \\
\hline Collected Oocytes & $9.2 \pm 5.6$ \\
\hline Mature Oocytes & $7.5 \pm 5.0$ \\
\hline
\end{tabular}

${ }^{\circ}$ Bologna criteria, ESHRE 2011

Patient characteristics are summarized in Table 1. The delay between surgery and IVF attempt was $3.3 \pm 3.8$ years. There was no significant difference in the number of more than $14 \mathrm{~mm}$ recruited follicles: $4.2 \pm 2.5$ on the operated side vs $4.1 \pm 2.4$ control side $(p=0.85)$. There was no significant difference for 10 to $14 \mathrm{~mm}$ recruited follicles between the groups ( $3.1 \pm 3.6$ vs $2.6 \pm 2.9$, $\mathrm{p}=0.46)$, for the total amount of recruited follicles $(7.2 \pm 4.9$ vs $6.7 \pm 4.1 ; \mathrm{p}=0.52)$, or for the follicular antral count $(8.3 \pm 4.7 \mathrm{vs}$ $7.9 \pm 5.3, \mathrm{p}=0.79$ ) (Table 2).

\section{Discussion}

In our study, there was no significant difference in the ovarian sonographic response during IVF protocol among patients treated by unilateral salpingectomy for EP.

\section{Table 2}

Sonographic Follicular Response during IVF Stimulation, comparing operated side versus control side, after unilateral salpingectomy.

\begin{tabular}{llll}
\hline & $\begin{array}{c}\text { Operated Side } \\
\text { Average +/- SD }\end{array}$ & Control Side & $\mathbf{P}^{\circ}$ \\
\hline $\begin{array}{c}\text { Antral Follicles (46 } \\
\text { patients) }\end{array}$ & $\mathbf{8 . 3} \pm 4.7$ & $\mathbf{7 . 9} \pm 5.3$ & $\mathbf{0 . 7 9}$ \\
$\begin{array}{c}\mathbf{1 5} \text { mm Follicles (55 } \\
\text { patients) }\end{array}$ & $\mathbf{4 . 2} \pm 2.5$ & $\mathbf{4 . 1} \pm 2.4$ & 0.85 \\
$\begin{array}{l}\mathbf{1 0} \text { to } \mathbf{1 4} \text { mm Follicles } \\
\quad \text { (55 patients) }\end{array}$ & $\mathbf{3 . 1} \pm 3.6$ & $\mathbf{2 . 6} \pm 2.9$ & 0.52 \\
$\begin{array}{c}\text { Total Follicles (55 } \\
\text { patients) }\end{array}$ & $\mathbf{7 . 2} \pm 4.9$ & $\mathbf{6 . 7} \pm 4.1$ & 0.52 \\
\hline
\end{tabular}

\footnotetext{
- Student Test
} 
Some limitations exist. The study does not allow the evaluation of the number of oocytes of each ovary, and thus, the evaluation of the final result of the IVF attempt. Data concerning follicular antral count for each ovary were available only for 46 patients (86\%). All the other ovarian reserve parameters were available for all patients. Another limitation concerns surgery data. Indeed, some details of the surgery were not accessible, including the level of expertise of the surgeon, tools used, tubal condition at surgery time, and the safeguarding of the mesosalpinx. Surgical approach (laparoscopy or laparotomy) was available for 19 of 55 patients (35\%). however, the strict inclusion of only patients treated by salpingectomy for EP generates a uniform group and a close surgical technique.

However, our study provides a contribution to the existing literature. The aim was to evaluate sonographic follicular response. Few studies have used this evaluation criterion [10-13]. Sonographic data were not used in the latest meta-analyses [8,14], and this limitation has been highlighted. This was a case-control study comparing the ovarian response within one patient (self-control study) and avoiding confounding factors that might exist between cases and controls (environmental impact, age, genetic, BMI, toxins, etc.).

Most of the accessible studies analyse heterogeneous populations in terms of surgical technique (salpingectomy, salpingotomy, and tubal exclusion, etc.) and its indication (EP, hydrosalpinx, endometriosis, and sterilisation, etc.), including Yoon's meta-analysis (patients treated by salpingectomy for EP of hydrosalpinx) and studies by Fan, Noventa and Kotlyar (salpingectomy for any indication, comparing uni- or bilateral surgery to a control population or self-control study) $[8,9,14,15]$. Most of the studies evaluated small samples, with multiple evaluation criteria (mature follicle, retrieved oocytes, obtained embryos, pregnancy rates, and ovarian reserve parameters). The use of pregnancy rates or obtained embryos is debatable criteria because they are dependent on many other ovarian reserve parameters (age, toxins, endometrial parameters, and father's parameters).

The punctured oocyte number only evaluates the oocyte puncture results. This number depends on the ovarian response but also on the puncture gesture, which could be impaired in the case of salpingectomy (post-surgery adhesions, modification of the position of the ovary and accessibility, etc.). Thus the ovarian evaluation, thanks to the study of sonographicrecruited follicles during stimulation, could be a good representation of global ovarian function and reserve. In 2012, Mutlu found that the most reliable ovarian reserve parameter was the antral follicle count and, thus, a sonographic parameter [16]. The sonographic monitoring of ovarian response could be a good evaluation of ovarian function and could allow a different approach from published studies.

In our study, the inclusion of patients who have all been treated by salpingectomy for EP permitted us to obtain a uniform population and a close surgical technique. Salpingectomy is probably the most invasive technique, in an emergency and tubal suffering context, which would have the most important impact on the ovarian function, as Kotlyan's study suggests it does [9]. Our population was quite important (55 patients) and is the second population with comparable patients available in the literature to this day, after Xi's study (76 cases) [11]. The delay between surgery and IVF attempt was $3.3 \pm 3.8$ years, quite a long period that permits the avoidance of confounding factors involved in early post-operative and post-ectopic pregnancy (inflammation, healing process, and ovarian collateral vascularization development, etc.). Only the first IVF attempt after surgery was studied to avoid bias induced by an overrepresentation of women having had several cycles, who present with poorer prognosis, statistically, and an overrepresentation of women having or not having a different ovarian response on the case or control side.

Our results are concordant with those found in studies by Dar, Tal and Xi [10,11,17]. In 2002, Tal did not find a significant difference in the ovarian response in 26 patients treated by salpingectomy for EP when compared to a control group of patients treated for male infertility (number of follicles, obtained oocytes and embryos). In 2012, Xi found an increase in the necessary gonadotrophin dose after salpingectomy but no difference in the number of mature follicles or obtained oocytes in a population of 76 operated patients (before and after salpingectomy) and 80 control patients. In 2000, in a population of 26 patients treated by unilateral salpingectomy for EP, Dar found no ovarian response reduction during IVF when comparing before vs. after surgery or case vs. control side (length of stimulation, gonadotrophin dose or obtained oocytes).

However, it is concerning that these publications contrast with some others that found a decrease in ovarian response. Indeed, Lass in 1998 showed a decrease in the number of follicles and oocytes ipsilateral to the surgery ovary in 29 patients treated by unilateral salpingectomy for EP [18]. There was no significant difference in the total number of follicles and oocytes obtained during the stimulation in comparison to a control group of 73 patients.

Although the results of our study and some of the other available studies in the literature are reassuring, the complete safety of this procedure is unsure, especially concerning the longterm consequences on ovarian function. Furthermore, a possible decrease in ovarian response to IVF stimulation would not necessarily mean a decrease in spontaneous fertility; just like the decrease in $\mathrm{AMH}$ is not linked to spontaneous time to pregnancy [19]. Nevertheless, caution concerning indications and the quality of tubal surgery is important, all the more concerning infertile women. Surgeon sensitivity to fertility problems is necessary [20]. Surgical technique standardization would allow stronger studies and the optimization of practices. Finally, patient information about risks and benefits of techniques is important to make an informed choice.

\section{Conclusion}

In our population, there was no significant difference in the sonographic ovarian response to IVF stimulation between the ovary on the operated side and the control ovary among patients treated by unilateral salpingectomy for ectopic pregnancy. More comparative studies on larger samples with reliable methodology and a standardized population are necessary to ensure the safety of this common surgery.

\section{References}

[1] Clausen I. Conservaive versus radical surgery for tubal pregnancy. A review. Acta Obstet Gynecol Scand 1996;75:8-12.

[2] Yao M, Tulandi T. Current status of surgical and nonsurgical management of ectopic pregnancy. Feril Steril 1997;67:421-33.

[3] Katz E, Akman MA, Damewood MD, García JE. Deleterious effect of the presence of hydrosalpinx on implantation and pregnancy rates with in vitro fertilization. Feril Steril 1996;66:122-5.

[4] Kontoravdis A, et al. Proximal tubal occlusion and salpingectomy result in similar improvement in in vitro fertilization outcome in patients with hydrosalpinx. Feril Steril 2006;86:1642-9.

[5] Zeyneloglu HB. Hydrosalpinx and assisted reproduction: options and rationale for treatment. Curr Opin Obstet Gynecol 2001;13:281-6.

[6] Johnson NP, Mak W, Sowter MC. Laparoscopic salpingectomy for women with hydrosalpinges enhances the success of IVF: a cochrane review. Hum Reprod Oxf Engl 2002;17:543-8.

[7] Beyth Y, Meiri U. Ferility following excision of the mesotubarium superius in the rabbit. Isr J Med Sci 1982;18:519-21.

[8] Fan M, Ma L. Effect of salpingectomy on ovarian response to hypersimulaion during in vitro fertilization: a meta-analysis. Feril Steril 2016;106:322-9 e9. 
[9] Kotlyar A, Gingold J, Shue S, Falcone T. The Effect of Salpingectomy on Ovarian Function. J Minim Invasive Gynecol 2017;24:563-78.

[10] Tal J, et al. Ovarian response to gonadotropin simulation in repeated IVF cycles after unilateral salpingectomy. J Assist Reprod Genet 2002:19:451-5.

[11] Xi W, Gong F, Tang Y, Zhang H, Lu G. Ovarian response to gonadotropins after laparoscopic salpingectomy for ectopic pregnancy. Int J Gynaecol Obstet Organ Int Fed Gynaecol Obstet 2012;116:93-6.

[12] Gelbaya TA, et al. Ovarian response to gonadotropins after laparoscopic salpingectomy or the division of fallopian tubes for hydrosalpinges. Feril Steril 2006;85:1464-8.

[13] Orvieto R, et al. Does salpingectomy affect the ipsilateral ovarian response to gonadotropin during in vitro fertilization-embryo transfer cycles? Feril Steril 2011;95:1842-4.

[14] Yoon S-H, et al. Does salpingectomy have a deleterious impact on ovarian response in in vitro fertilization cycles? Feril Steril 2016;106:1083-92 e5.
[15] Noventa M, et al. Salpingectomy before assisted reproductive technologies: a systematic literature review. J Ovarian Res 2016;9:74.

[16] Mutlu MF, et al. Antral follicle count determines poor ovarian response better than aniMüllerian hormone but age is the only predictor for live birth in in vitro fertilization cycles. J Assist Reprod Genet 2013;30:657-65.

[17] Dar P, Sachs GS, Strassburger D, Bukovsky I, Arieli S. Ovarian function before and after salpingectomy in artificial reproductive technology patients. Hum Reprod Oxf Engl 2000;15:142-4.

[18] Lass A, et al. Effect of salpingectomy on ovarian response to superovulaion in an in vitro ferilization-embryo transfer program. Feril Steril 1998;70:1035-8.

[19] Streuli I, et al. AMH concentration is not related to effective time to pregnancy in women who conceive naturally. Reprod Biomed Online 2014;28:216-24.

[20] Kalra GS, Campbell S, Nargund G. Ovarian reserve may be compromised after adnexal surgery: Are we sufficiently fertility- focused in our surgical training? Facts Views Vis ObGyn 2016;8:104-8. 